\title{
Construction and Validation of a Novel Pyroptosis-Related Gene Signature to Predict the Prognosis of Uveal Melanoma
}

\section{OPEN ACCESS}

Edited by:

Tugba Bagci-Onder,

Koç University, Turkey

Reviewed by:

Ibrahim Kulac,

Koç University, Turkey

Xingyu Chen,

Central South University, China

$\mathrm{Li} \mathrm{Li}$,

Peking Union Medical College, China

${ }^{*}$ Correspondence: Ming Wang wangming@jsph.org.cn

${ }^{\dagger}$ These authors share first authorship

Specialty section:

This article was submitted to Cell Death and Survival, a section of the journal Frontiers in Cell and Developmental

Biology

Received: 19 August 2021 Accepted: 05 November 2021 Published: 26 November 2021

Citation:

Cao Y, Xie J, Chen L, Hu Y, Zhai L, Yuan J, Suo L, Shen Y, Ye R, Li J, Gong Z, Dong Y, Bao W, Li H and Wang $M$ (2021) Construction and Validation of a Novel PyroptosisRelated Gene Signature to Predict the

Prognosis of Uveal Melanoma. Front. Cell Dev. Biol. 9:761350. doi: 10.3389/fcell.2021.761350

\begin{abstract}
Yuan $\mathrm{Cao}^{1 \dagger}$, Jiaheng Xie ${ }^{2 \dagger}$, Liang Chen ${ }^{3 \dagger}$, Yiming $\mathrm{Hu}^{4}$, Leili Zhai ${ }^{1}$, Jin Yuan ${ }^{1}$, Long Suo ${ }^{1}$, Yaming Shen ${ }^{1}$, Rong Ye ${ }^{1}$, Jiajun $\mathrm{Li}^{1}$, Zixuan Gong ${ }^{5}$, Yunfan Dong ${ }^{6}$, Wei Bao ${ }^{7}$, Huan $\mathrm{Li}^{1}$ and Ming Wang ${ }^{2 *}$

${ }^{1}$ The Fourth Clinical Medical College, Nanjing Medical University, Nanjing, China, ${ }^{2}$ Department of Burn and Plastic Surgery, The First Affiliated Hospital of Naniing Medical University, Nanjing, China, ${ }^{3}$ Department of General Surgery, Fuyang Hospital Affiliated to Anhui Medical University, Fuyang, China, ${ }^{4}$ College of Pharmacy, Jiangsu Ocean University, Lianyungang, China, ${ }^{5}$ Urology Department, The First Affiliated Hospital of Nanjing Medical University, Nanjing, China, ${ }^{6}$ Department of Gastroenterology, Nanjing First Hospital, Nanjing Medical University, Nanjing, China, ${ }^{7}$ Urology Department, The Second Affiliated Hospital of Nanjing Medical University, Nanjing, China
\end{abstract}

Uveal melanoma is the most common primary intraocular tumor with a poor prognosis. Currently, treatment for UVM is limited, and the development of drug resistance and tumor recurrence are common. Therefore, it is important to identify new prognostic biomarkers of UVM and explore their role in the tumor microenvironment. Pyroptosis is a way of cell programmed death, and related research is in full throttle. However, the role of pyroptosis in UVM is unclear. In this study, we constructed the prognosis model of pyroptosis-related genes of UVM. This model can accurately guide the prognosis of UVM, and different groups differ in immune infiltration. We further verified our results in cell experiments. To some extent, our study can provide new ideas for the diagnosis and treatment of UVM.

Keywords: uveal melanoma, pyroptosis, signature, immune microenvironment, programmed cell death

\section{INTRODUCTION}

Uveal melanoma is a general term for melanoma that occurs in the choroid, iris, and ciliary body (Chattopadhyay et al., 2016; Kaliki and Shields, 2017). The number of new UVM cases in the United States is 1,500 per year (Smit et al., 2020). Although the total number of cases is small, UVM is the most common primary intraocular malignancy whose most common clinical manifestation is painless vision loss (Luke et al., 2020). When the tumor is large, serous retinal detachment is often associated (Kaliki and Shields, 2017). Once metastasis has occurred, the prognosis for UVM is often poor and treatment options are limited (Carvajal et al., 2018). Treatment options for advanced cutaneous melanoma, such as targeted therapy and immunotherapy, seem to be attractive options for advanced UVM (Jager et al., 2020). Although these treatments are often effective, these treatments are also limited (Jager et al., 2020). It is because that a significant proportion of UVM patients develop drug resistance during treatment, which often leads to tumor recurrence and patient death. Hence, it is time to look for new prognostic indicators of UVM and explore its implications for cancer treatment.

Recently, programmed cell death has become a hot topic in biology (Kovacs and Miao, 2017; Chen et al., 2021a). Pyroptosis is a mode of programmed cell death that plays an important role in homeostasis regulation as well as disease occurrence (Tang et al., 2020; Chen et al., 2021b; Chen et al., 2021c). Pyroptosis is a gasdermin (GSDM) dependent process of membrane perforation 
accompanied by exudation of inflammatory contents (Xie et al., 2021a). In cancer, pyroptosis has a dual effect. On the one hand, we can reduce tumor load by inducing cancer cells pyroptosis (Xia et al., 2019; Ruan et al., 2020); On the other hand, inflammatory substances released by pyroptosis are involved in the formation of the tumor microenvironment (Jiang et al., 2020). Our current understanding of pyroptosis is far from sufficient, especially in UVM. Pyroptosis is a promising research field.

Here, we combine bioinformatics with cellular experiments to provide a prognostic signature of genes associated with pyroptosis for UVM. Our findings can provide some new ideas for the diagnosis and treatment of UVM.

\section{MATERIALS AND METHODS}

\section{Information Extraction of Datasets}

We downloaded uveal melanoma RNA sequencing data from TCGA database (https://portal.gdc.cancer.gov/). Data inclusion criteria were: 1) patients diagnosed with uveal melanoma; 2) Patients had detailed mRNA expression and clinical information. Collectively, 80 patients met the inclusion criteria, the gene expression of whom were downloaded for further analysis. (Those with a follow-up of fewer than 30 days were excluded).

\section{Identification of Genes Associated With Cell Pyroptosis}

81 pyroptosis-related genes were extracted from GENECARDS (https://www.genecards.org/). Setting "pyroptosis" as a key word, we searched for pyroptosis-related protein coding genes in the Genecard database. Protein coding genes with correlation score $>0.6$ were included in our subsequent analysis. A total of 81 genes were eventually included in our analysis.

\section{Identification of Prognostic Pyroptosis-Related Genes}

We initially performed a univariate Cox regression to screen for those potentially prognostic pyroptosis-related genes derived from the TCGA data using $\mathrm{R}$ software (version 4.1.0). Packages "survival" were utilized for cox regression analysis. Using R packages "glmnet", Lasso regression was subsequently performed with those genes that are significantly correlated with patient survival (with $p<0.05$ ).

Risk scores were calculated based on the LASSO regression results. The scoring formula is: risk score $=\sum_{i=1}^{n} \beta_{i}^{*}$ (expression of pyroptosis associated gene $e_{i}$. Using a median risk score, patients were divided into high-risk and low-risk groups. The defined groups were respectively analyzed during further studies.

\section{Construction of the Prognostic Model}

After the division of patients into high-risk and low-risk groups, survival analysis was performed for both groups to identify the prognostic value of the model. Survival analysis was performed within various subgroups, such as gender, age, and conditions of tumor staging. The accuracy of the established prognosis model was verified by the calibration curve and ROC curve on 1,3 , and 5 years basis.

\section{Clinical Prediction Value of the Established Prognostic Model}

We used univariate and multivariate Cox regressions, respectively, to test whether risk score and clinical characteristics (age, sex, stage) were effective prognostic indicators for uveal melanoma patients. Using $\mathrm{R}$ package "forestplot", the results of cox regression were visualized to see whether the risk score model we previously established was an independent prognostic factor.

\section{Gene Ontology and Kyoto Encyclopedia of Genes and Genomes Analysis}

Differential genes from high-risk and low-risk groups were then subjected to Gene Ontology (GO) and Kyoto Encyclopedia of Genes and Genomes (KEGG) enrichment analysis using $\mathrm{R}$ packages "ClusterProfiler" (version 3.0.4), the significantly enriched pathways and ontologies and the associated genes of which were illustrated. Gene Set Enrichment analysis (GSEA) was used as the next enrichment analysis. GSEA required a preexisting set of biologically significant genes (like genes in a pathway), and then the genes in the set (with the same meaning/function) were calculated and summarized into a single enrichment score. This analytical approach added to the interpretability and was used in this study to assess changes in the activity of the pathway/function of the gene set and to select the gene set with $p<0.05$. Gene Set Variation Analysis (GSVA) was one of the GSEA algorithms. In this study, we collected genes related to immune function and obtained the score of each sample's immune function Gene Set by GSVA calculation.

\section{Analysis of Immune Microenvironment}

By enquiry into TIMER database (http://timer.cistrome.org/), we downloaded 7 kinds of algorithms of each patient's immune infiltration situation. Then we analyzed the expression of immune cells in the high and low-risk groups, and isolated the cells with differential expression $(p<0.05)$, before developing a heatmap, At the same time, we studied the expression of immune checkpoint genes between the high and low-risk groups. Similarly, immune checkpoint genes with different expressions $(p<0.05)$ were extracted and the boxplot was made. In DREIMT database (http:// www.dreimt.org/), we further analyzed the correlation between the model and immune cells by inputting 80 down-regulated genes and the first 199 up-regulated genes in ascending order of $p$-value into the website. Patients with UVM were scored using the "ESTIMATE" R package to obtain tumor purity score, immune score, stromal score, and total score for each patient.

\section{Potential Drug Candidate Prediction Using R Software and the Construction of a Nomogram}

Using the "pRRophetic" package and the expression matrix of gastric cancer patients, we predicted the minimum drug 
inhibition concentration $\left(\mathrm{IC}_{50}\right)$ of drugs in uveal melanoma patients of high-risk and low-risk groups, and finally obtained drugs that have statistically different $\mathrm{IC}_{50}$ values and may become candidates for the treatment of uveal melanoma. A nomogram of the patient "TCGA-VD-A8KH" was plotted using the "Regplot" package to integrate risk groups with clinical features.

\section{Cell Culture and Transfection}

The human invasive uveal melanoma cell line (MuM2B) was purchased from Fuheng Biology Inc. (Fuheng, Shanghai, China). MUM2B cells were cultured in Roswell Park Memorial Institute 1,640 (RPMI1640, Gibco, Carlsbad, CA, United States), supplemented with 10\% Fetal Bovine Serum (Gibco), along with $100 \mathrm{U} / \mathrm{mL}$ penicillin and $100 \mu \mathrm{g} / \mathrm{ml}$ streptomycin (Gibco). The cells were cultured in an atmosphere of $5 \% \mathrm{CO}_{2}$ and at a temperature of $37^{\circ} \mathrm{C} .24 \mathrm{~h}$ prior to transfection, MUM2Bs were seeded onto six-well tissue culture plates at a density of 50,000 cells per well. Cells were starved in $0.5 \%$ FBS medium for $6 \mathrm{~h}$ before any further treatment.

The small interfering RNA (siRNA) probe for ANO6 and GAPDH positive control oligonucleotides and its control siRNAs were synthesized by Shanghai GenePharma Inc. (Shanghai, China). The sequence of the siRNAs were illustrated in (Supplementary Table S1). Lipo6000 ${ }^{\mathrm{TM}}$ Transfection reagent (Beyotime, Nanjing, China) was used for the transfection of siRNA according to the manufacturer's instructions.

\section{Quantitative Real-Time Polymerase Chain Reaction (qRT-PCR)}

Total cellular RNAs were isolated from cells using Trizol Reagent (Invitrogen, Carlsbad, CA, United States) according to the manufacturer's instructions. The reverse transcription was conducted using the reverse transcription kit provided by Takara (Otsu, Shiga, Japan). Real-time polymerase chain reaction (RT-PCR) was performed using a QuantiTect SYBR Green PCR Kit (Takara), and on a Applied Biosystems QuantStudio 1 (Thermo, Waltham, MA, United States). Relative quantification was determined using the $-2 \Delta \Delta \mathrm{Ct}$ method. The relative expression of messenger RNA (mRNA) for each gene was normalized to the level of glyceraldehyde-3phosphate dehydrogenase (GAPDH) mRNA. The primers were synthesized by GenePharma Inc. (Shanghai, China), the sequence of which were listed in (Supplementary Table S2).

\section{Cell Proliferation and Cell Cycle Analysis}

5-ethynyl-2 deoxyuridine (EdU) assay was performed according to the manufacturer's instructions (Beyotime, Shanghai, China), MUM2B cells were incubated with EdU for $2 \mathrm{~h}$. The number of proliferating cells were analyzed under an Olympus confocal microscope (Olympus, Tokyo, Japan).

\section{Cell Migration Assay}

A scratch wound-healing migration assay was performed in transfected and non-transfected uveal melanoma cell lines to validate the relationship between the prognostic genes and the tumor cell migration ability. When the MUM2Bs reached
90-100\% confluence in the 6-well culture plate, cells were subjected to serum-free RPMI1640 medium for $24 \mathrm{~h}$. After serum starvation, one line within the MUM2Bs were scraped using a sterile plastic pipette tip in each cultured well. The cells were washed twice in warm serum-free medium to remove cellular debris After 0,6 , and $12 \mathrm{~h}$ the scratch wounds were subjected to microscope photography. Images were acquired using a microscope (Olympus, Tokyo, Japan), and cell migration was determined by the percentage of the wound closure area in five independent experiments using the Image J software.

\section{Data Processing and Statistical Analysis}

The quantification of the experiment data was conducted using Image J software (version 1.0.3). All experimental data were analyzed using IBM SPSS software (version 16.0.0) multiple comparisons were conducted using one-way ANOVA and Student- Newman-Keuls (SNK) multiple comparison method, and $p<0.05$ was considered statistically significant.

\section{RESULTS}

\section{Pyroptosis-Related Genes Were Identified to Calculate the Risk Score}

The flow diagram of our present study is illustrated in Figure 1. Based on the previously described inclusion criteria, $80 \mathrm{UVM}$ patients were treated as the training cohort. Using univariate Cox regression, we screened for the genes which are potentially related to the patient's prognosis (with $p$-value $<0.05$ ), both in the training cohort and in the validation cohort as well. An intersection was performed subsequently to achieve a higher confidence level, which included nine potentially prognostic genes. The forestplot which contained the Hazard Ratio (HR) and its 95\% confidence interval can be seen in Figure 2A. The potential prognostic genes were subsequently subjected to LASSO-Cox regression in the training cohort to generate a Prognostic signature (Figures 2B,C). 5 pyroptosis-related genes with prognostic value were eventually obtained. The names and the coefficients of the prognostic genes were listed in Table1. The risk score was calculated according to the formula which was previously described.

\section{Based on the Median Risk Score, 80 Melanoma Patients in TCGA Were Divided Into High-Risk and Low-Risk Groups}

The group model based on risk score effectively predicted the prognosis of melanoma patients.

To further test the confidence of the risk model, a survival analysis was performed between high-risk group and low risk groups between the training cohort (Figure 3A) and the validation cohort (Figure 3B). Meanwhile, a further, more specifically classified survival analysis was performed in different subgroups in the training cohort. The survival rate of patients in the high-risk group suffered a more drastic decrease, regardless of the age, gender, and tumor stages. The differences 

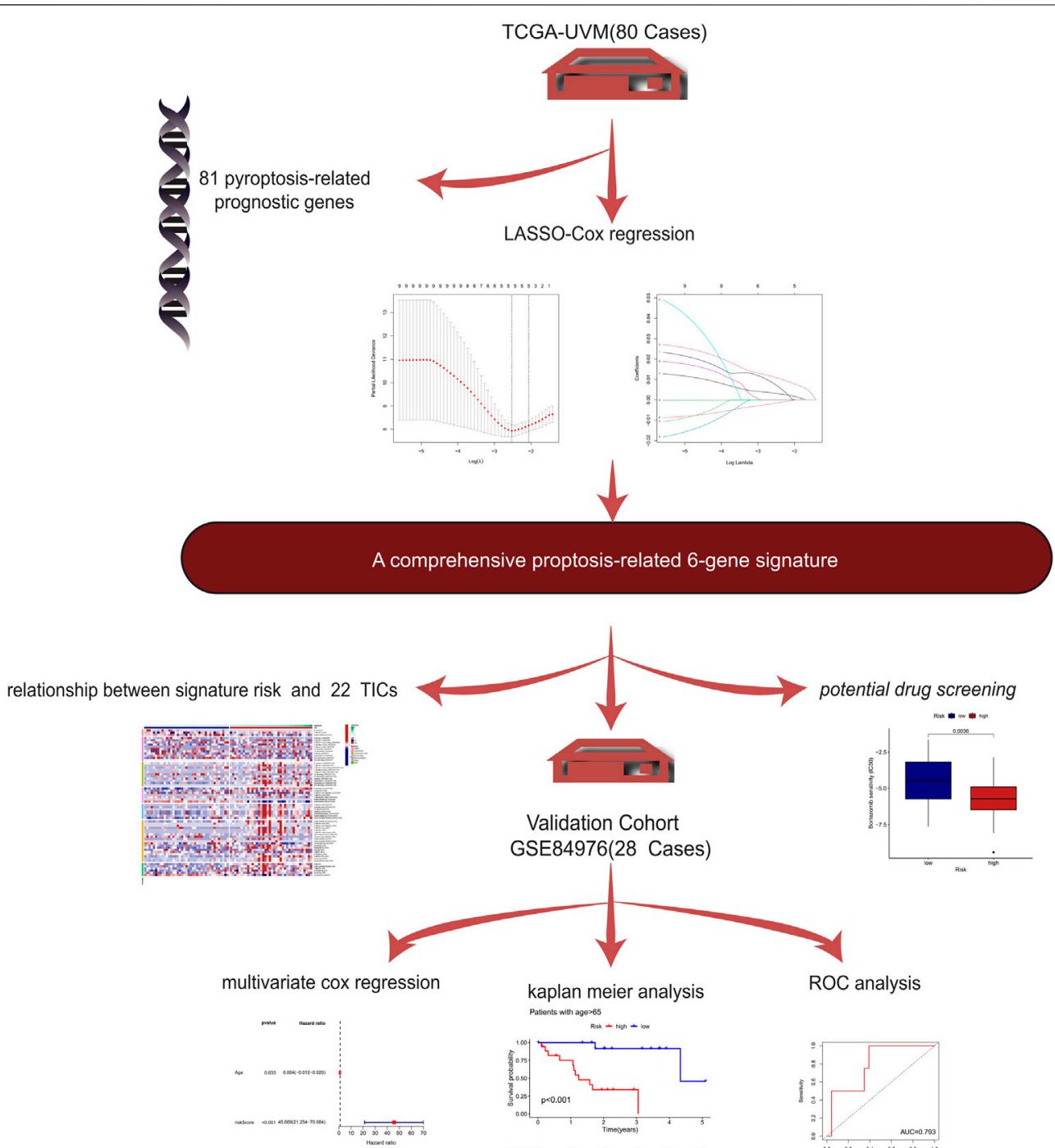

kaplan meier analysis

ROC analysis
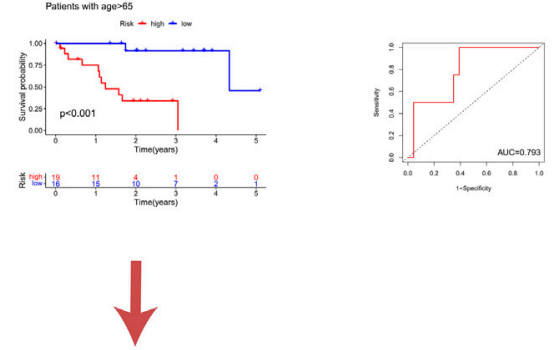

Experimental validation

\section{CInسm监}

siRNA knockdown of gene ANO6

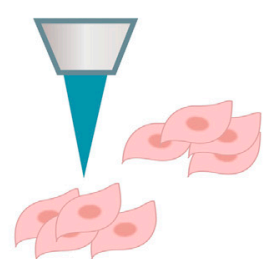

wound healing assay

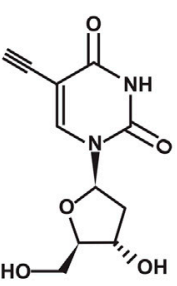

EdU assay

FIGURE 1 | The flow chart.

were statistically significant $(p<0.001)$ (Figures Figure3C-J). The ROC curve of patient's survival of different years in the training cohort and the validation cohort showed that the model had a potent predicting ability, with the 1-year, 2-years, 3-years and 5 -years AUC being $0.79,0.83,0.854,0.886$ respectively in the training cohort, and the 2-years, 3-years, and 5-years AUC being $0.856,0.846,0.873$ respectively in the validation cohort. (Figures 4A,B). The findings revealed that the model was effective in predicting patient's survival. The distribution of the risk scores, outcome status, and gene profiles of the gene signature in the 


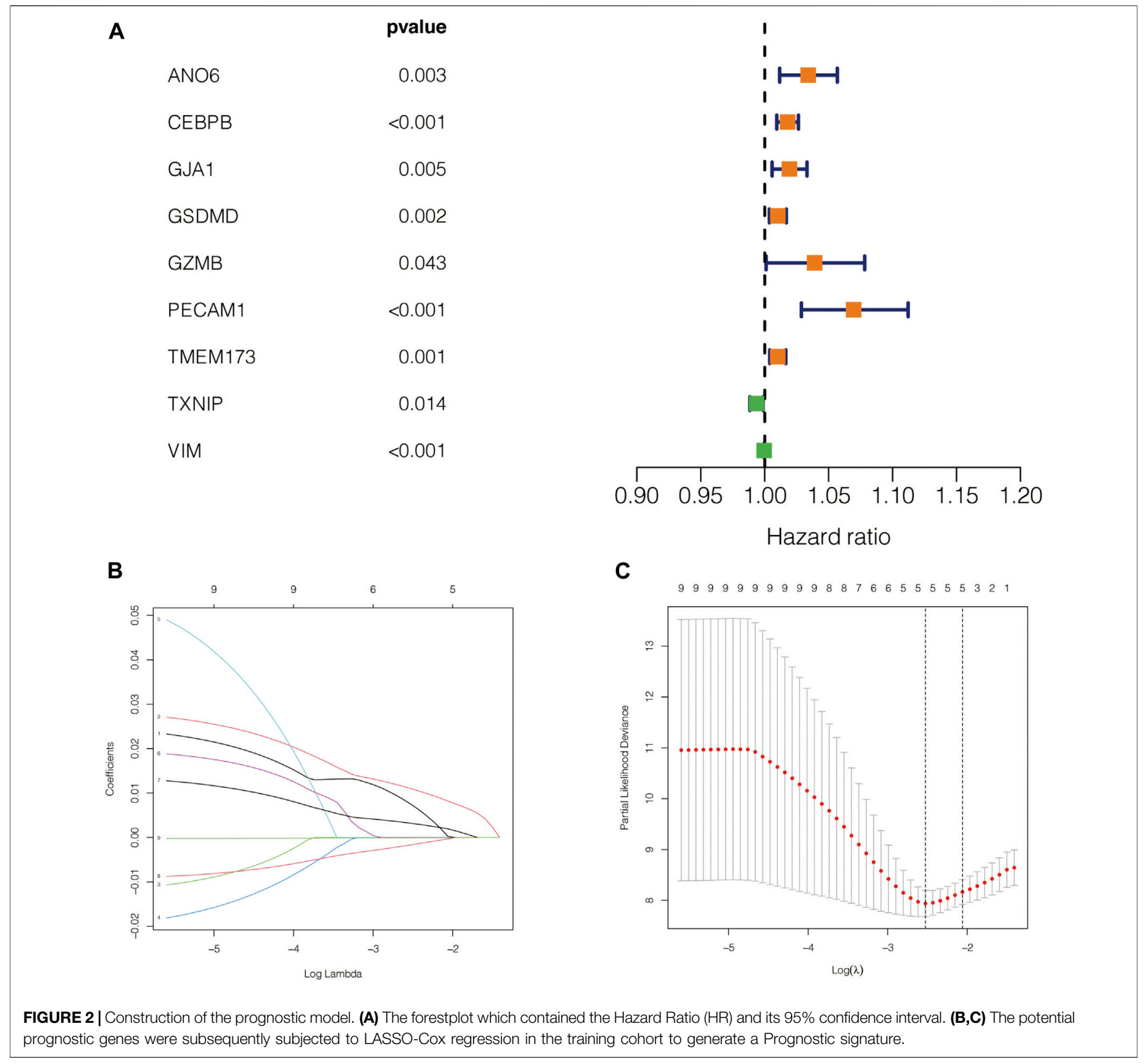

TABLE 1 | The names and the coefficients of the prognostic genes.

Gene

ANO6

CEBPB

TMEM173

TXNIP

VIM

training and validation cohort were shown in Figures 4C,D. The risk group successfully predicted the outcomes of the patients in both the training cohort and the validation cohort, with significantly more events found in the high-risk group.
Moreover, the expression of genes ANO6, CEBPB and TMEM173 were upregulated, while genes VIM, TXNIP were seen downregulated in the high-risk group both in the training cohort and in the validation cohort, which was in accordance with our previous risk model (Figures 4C,D).

Univariate and Multivariate Cox Regression Revealed That the Risk Score Was an Independent Prognostic Factor in UVM

\section{Patients}

In order to explore whether risk score was an independent influencing factor for melanoma patients, univariate and multivariate Cox regression were performed for risk score, age, 


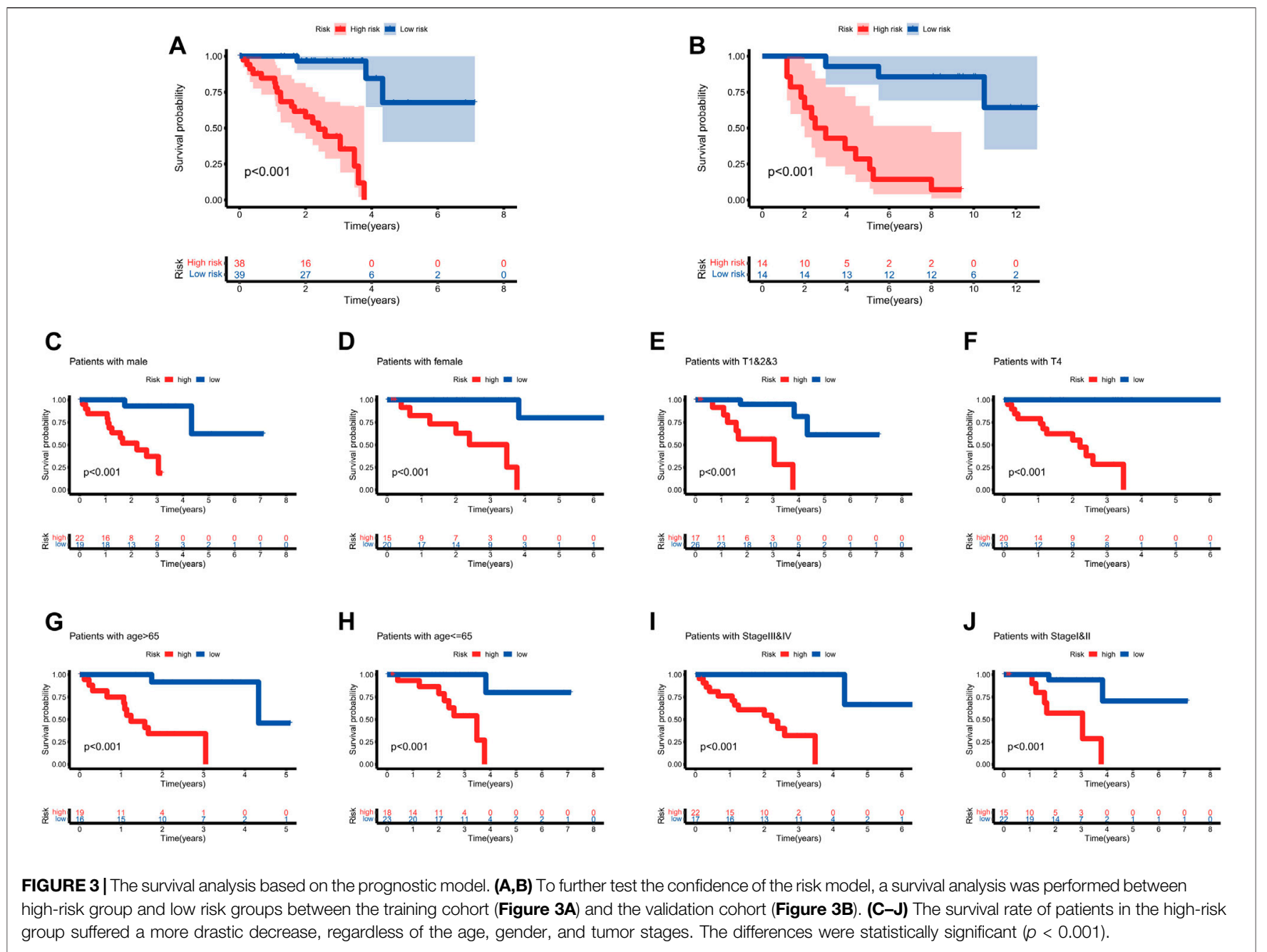

gender, TNM stage and other factors in both the training cohort and the validation cohort. Cox regression showed that risk score was a independent prognostic factor using univariate cox regression using both univariate and multivariate regression (Figures 5A,B), despite the fact that tumor stage could also be considered as an independent prognostic factor by means of univariate cox regression. Likewise, risk score was also an independent prognostic factor with statistical significance in validation cohort (Figures 5C,D).

\section{Gene Ontology and Kyoto Encyclopedia of Genes and Genomes Enrichment Analysis Revealed That the Differential Genes Between High Risk and Low Risk Groups Were Strongly Correlated With Immune Response}

GO Enrichment analysis revealed that, biological processes like T cell activation and differentiation, lymphocyte differentiation and leukocyte cell-cell adhesion; molecular functions such as chemokine activity, cytokine activity, immune receptor activity, as well as cytokine receptor activity were enriched in differential genes between the high- risk group and the low-risk group. The enriched genes were mainly concentrated in the external side of the plasma membrane, which was in line with our findings that the differential genes were correlated immune response. (Figures 6A,B). KEGG analysis revealed a more specific picture of which pathways were enriched between the two groups. Not only was Th1 and Th2 cell differentiation related to the risk model, but PD-1 and PD-L1 activity was also concerned (Figures 6C,D). GSEA showed that immune-related functional gene sets were mainly enriched in the high-risk group, including allograft rejection, IL6-JAK-STAT3 signaling pathway, inflammatory response, interferon-alpha response, interferon-gamma response (Figure 6E). Next, 2,483 immune-related genes were downloaded from IMMPORT database. Through GSEA analysis, we found that these immunerelated genes are mainly enriched in antigen processing and presentation, cell adhesion molecules, cytokine receptor interactions, and cytotoxicity mediated by natural killer cells in the high-risk group. Axon guidance was the main enrichment pathway in the low-risk group (Figure 6F).

\section{Analysis of Immune Microenvironment}

To further analyze the relationship between tumor immune response and the risk model we previously constructed, 

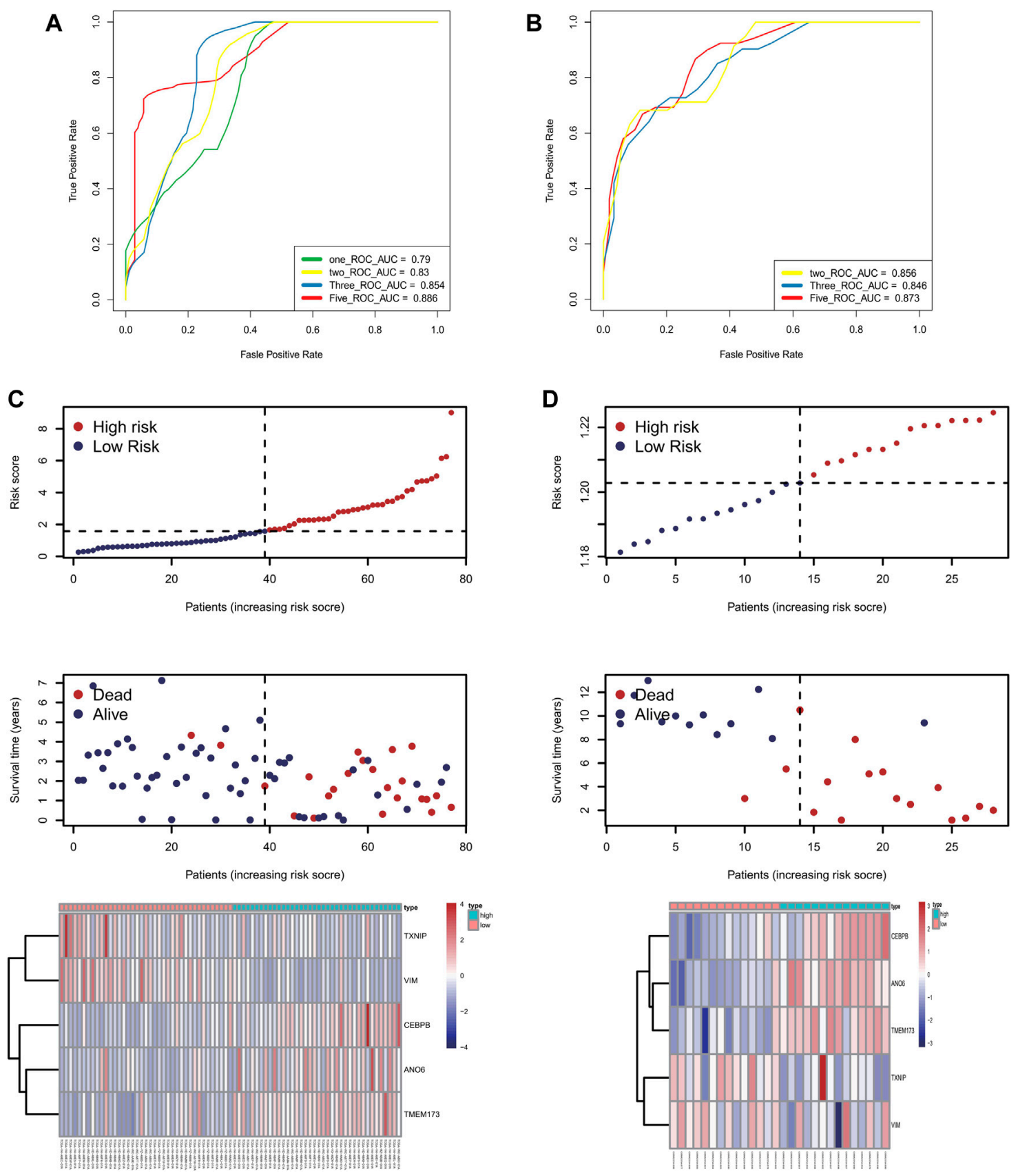

FIGURE 4 | The accuracy of this prognostic model. (A,B) The ROC curve of patient's survival of different years in the training cohort and the validation cohort showed that the model had a potent predicting ability, with the 1-year, 2-years, 3-years and 5-years AUC being $0.79,0.83,0.854,0.886$ respectively in the training cohort (Panel 4A), and the 2-years, 3-years and 5-years AUC being 0.856, 0.846, 0.873 respectively in the validation cohort (Panel 4B). (C,D) The distribution of the risk scores, outcome status, and gene profiles of the gene signature in the training and validation cohort were shown. The risk group successfully predicted the outcomes of the patients in both the training cohort and the validation cohort, with significantly more events found in the high-risk group. Moreover, the expression of genes ANO6, CEBPB and TMEM173 were upregulated, while genes VIM, TXNIP were seen downregulated in the high-risk group both in the training cohort and in the validation cohort, which was in accordance with our previous risk model.

immune infiltration analysis using various methods was conducted. The levels of immune infiltration differed between different risk groups. In Figure $\mathbf{7 A}$, the samples in the training cohort were displayed according to ascending risk scores, while the levels of immune infiltration in high-risk and low-risk groups were calculated by algorithm such as TIMER, CIBERSORT, Quantiseq and other methods, which were marked in different colors. The heatmap showed the level of each immune cell infiltration in the tumor microenvironment of the two groups of patients under different algorithms. We could see that the 


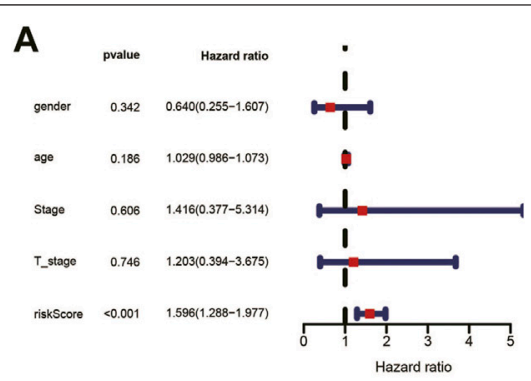

Multivariate Regression

C

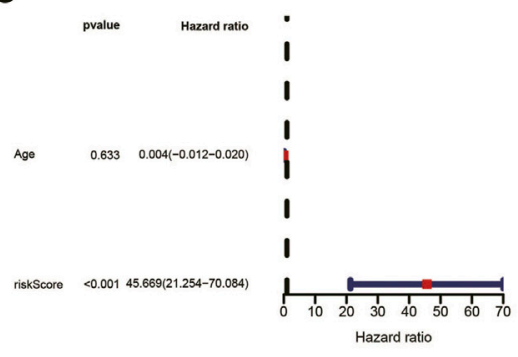

Multivariate Regression

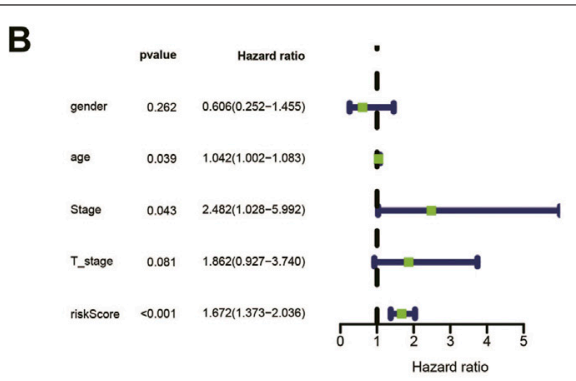

Univariate Regression

D

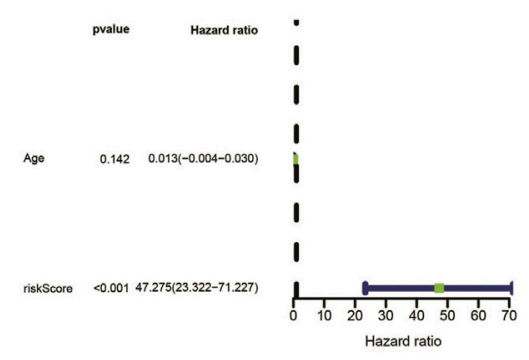

Univariate Regression

FIGURE 5 | Univariate and Multivariate Cox regression revealed that the risk score was an independent prognostic factor in UVM patients. (A,B) In order to explore whether risk score was an independent influencing factor for melanoma patients, univariate and multivariate Cox regression were performed for risk score, age, gender, TNM stage and other factors in both the training cohort and the validation cohort. Cox regression showed that risk score was a independent prognostic factor using univariate cox regression using both univariate (Panel $5 \mathrm{~A}$ ) and multivariate (Panel 5B) regression, despite the fact that tumor stage could also be considered as an independent prognostic factor by means of univariate cox regression. (C,D) Likewise, risk score was also an independent prognostic factor with statistical significance in validation cohort.

high-risk group tended to have higher levels of immune cells in the tumor microenvironment. Likewise, The immune checkpoint genes were expressed differently in the two groups and gene tended to be higher in the high-risk group $\left.{ }^{* * *} p<0.001\right)$ (Figure 7B). In Figures 8A-D, we can see the differences in stromal score, tumor purity, ESTIMATE score and total score between the two groups $\left({ }^{* *} p<0.001\right)$. The stromal score and ESTIMATE score were higher in the high-risk group than in the low-risk group $(p<0.001)$. Patients in the high-risk group had lower tumor purity scores than those in the low-risk group $(p<$ 0.001). In terms of total score, the score of patients in the highrisk group showed a higher trend.

\section{Analysis of Sensitivity Difference of Antitumor Drugs in Different Groups and the Construction of a Nomogram}

The study of the sensitivity of different groups of patients to antitumor drugs can provide help for the formulation of future treatment regimens. Boxplot showed the differential $\mathrm{IC}_{50}$ of our previously established high-risk and low risk groups in the TCGA cohort. The high-risk group was more sensitive to antitumor drugs like Rapamycin, Pazopanib, Bortezomib, Cisplatin, Methotrexate, Mitomycin. C, Bortezomib and Imatinib. Those antitumor drugs were potentially more capable of inhibiting high-risk uveal melanoma with relatively minor dosage. However, further investigations are required to understand their mechanisms in Uveal Melanoma inhibition. (Figures 9A-H). To further evaluate the survival of UVM patients, we drew a Nomogram combining the risk value and clinical characteristics of the model. As shown in Figure 10, we found that the 1-, 3-, and 5-years mortality rates of patient "TCGAVD-A8KH" were $0.00272,0.0339$ and 0.555 , respectively.

\section{ANO6 Knockdown Slows Down Uveal Melanoma Cell Proliferation in vitro}

We further performed the experimental analysis of genes that are in the prognostic signature to validate their functions in uveal melanoma cell growth and migration. Because gene ANO6 had a relatively higher level of hazard ratio, and tended to be robust in our previously constructed models, the oncogenic role of ANO6 was tested in further experiments. Firstly, RT-qPCR analysis was performed to validate the knockdown of gene ANO6 mRNA. Figure 11A showed that the level of ANO6 mRNA expression was significantly downregulated after ANO6 siRNA transfection in MUM2B cell lines, which is valid for further investigation $(p<0.001)$.

5-ethynyl-2 deoxyuridine (EdU) assay was performed to test whether the knockdown of gene ANO6 could influence uveal melanoma cell proliferation in vitro. After knockdown of ANO6 gene, the in MUM2B cell lines showed a significant decrease in 


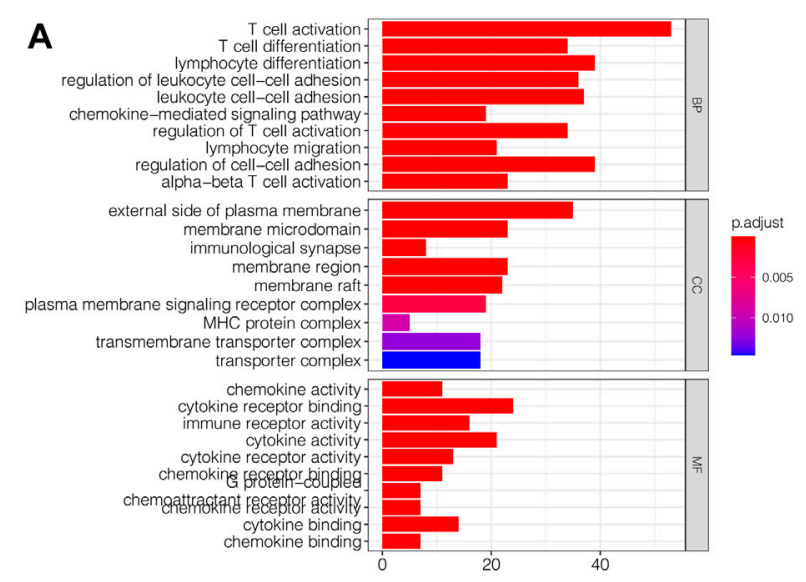

C Viral protein interaction with cytokine and cytokine receptor Cell adhesion molecules Th1 and Th2 cell differentiation Hematopoietic cell lineage Type I diabetes mellitu Primary immunodeficiency Th17 cell differentiatio Chemokine signaling pathway
Allograft rejection

T cell receptor signaling pathwa Graft-versus-host disease Inflammatory bowel disease NF-kappa B signaling pathway Antigen processing and presentation-
Natural killer cell mediated cytotoxicity Natural killer cell mediated cytotoxicity
Autoimmune thyroid disease Autoimmune thyroid disease Human T-cell leukemia virus 1 infection Rheumatoid arthritis

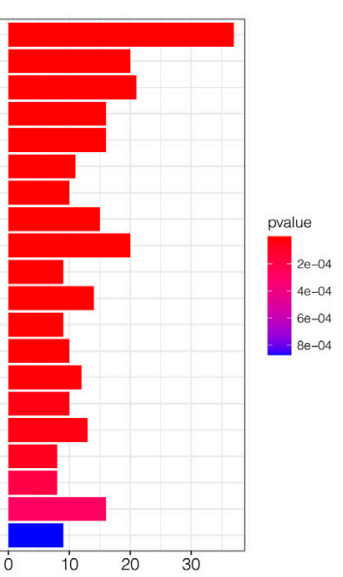

E

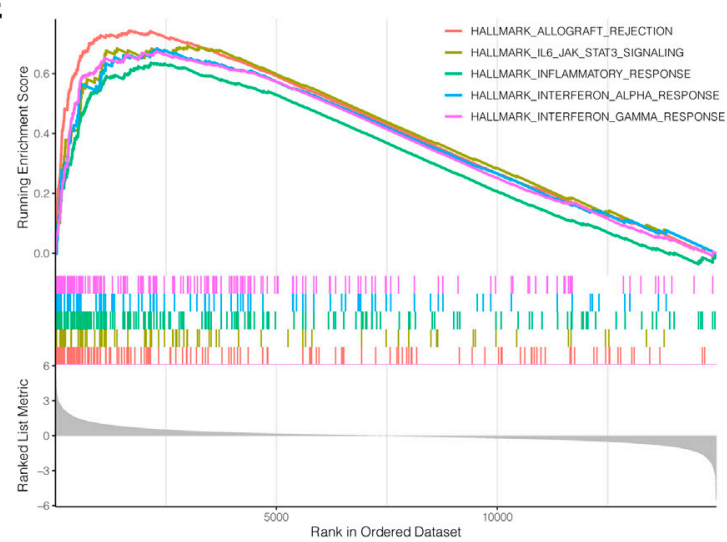

B
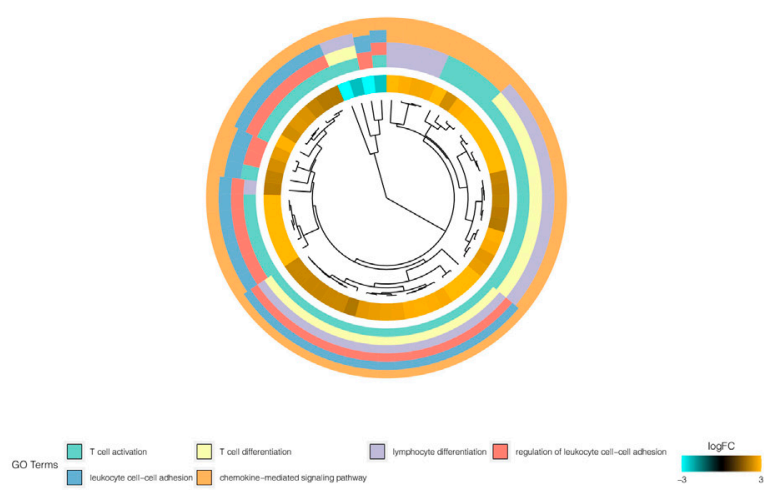

D

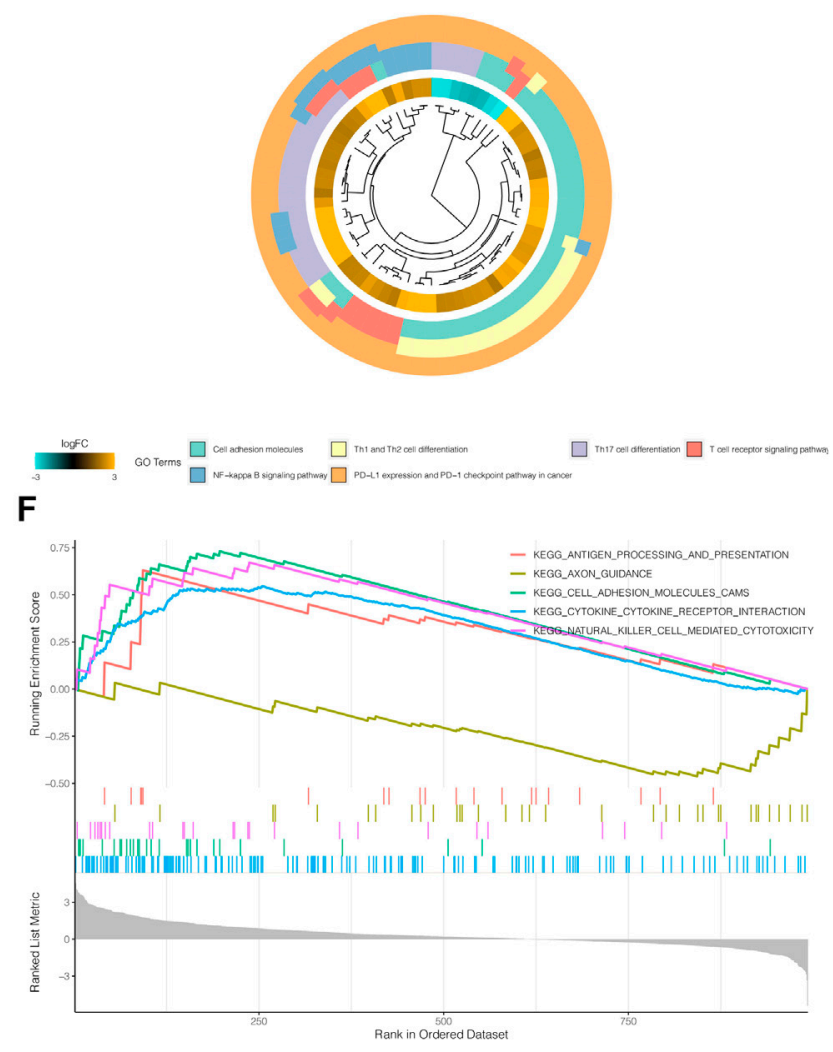

FIGURE 6 | GO and KEGG Enrichment Analysis revealed that the differential genes between high risk and low risk groups were strongly correlated with immune response. (A,B) GO Enrichment analysis revealed that, biological processes like T cell activation and differentiation, lymphocyte differentiation and leukocyte cell-cell adhesion; molecular functions such as chemokine activity, cytokine activity, immune receptor activity, as well as cytokine receptor activity were enriched in differential genes between the high-risk group and the low-risk group. The enriched genes were mainly concentrated in the external side of the plasma membrane, which was in line with our findings that the differential genes were correlated immune response. (C,D) KEGG analysis revealed a more specific picture of which pathways were enriched between the two groups. Not only was Th1 and Th2 cell differentiation related to the risk model, but PD-1 and PD-L1 activity was also concerned. However, further studied are still needed to validate the relationship between the relationship with our model and immune response. (E) GSEA showed that immune-related functional gene sets were mainly enriched in the high-risk group, including allograft rejection, IL6-JAK-STAT3 signaling pathway, inflammatory response, interferon-alpha response, interferon-gamma response. (F) 2,483 immune-related genes were downloaded from IMMPORT database. Through GSEA analysis, we found that these immune-related genes are mainly enriched in antigen processing and presentation, cell adhesion molecules, cytokine receptor interactions, and ccytotoxicity mediated by natural killer cells in the high-risk group. Axon guidance was the main enrichment pathway in the low-risk group. 
A

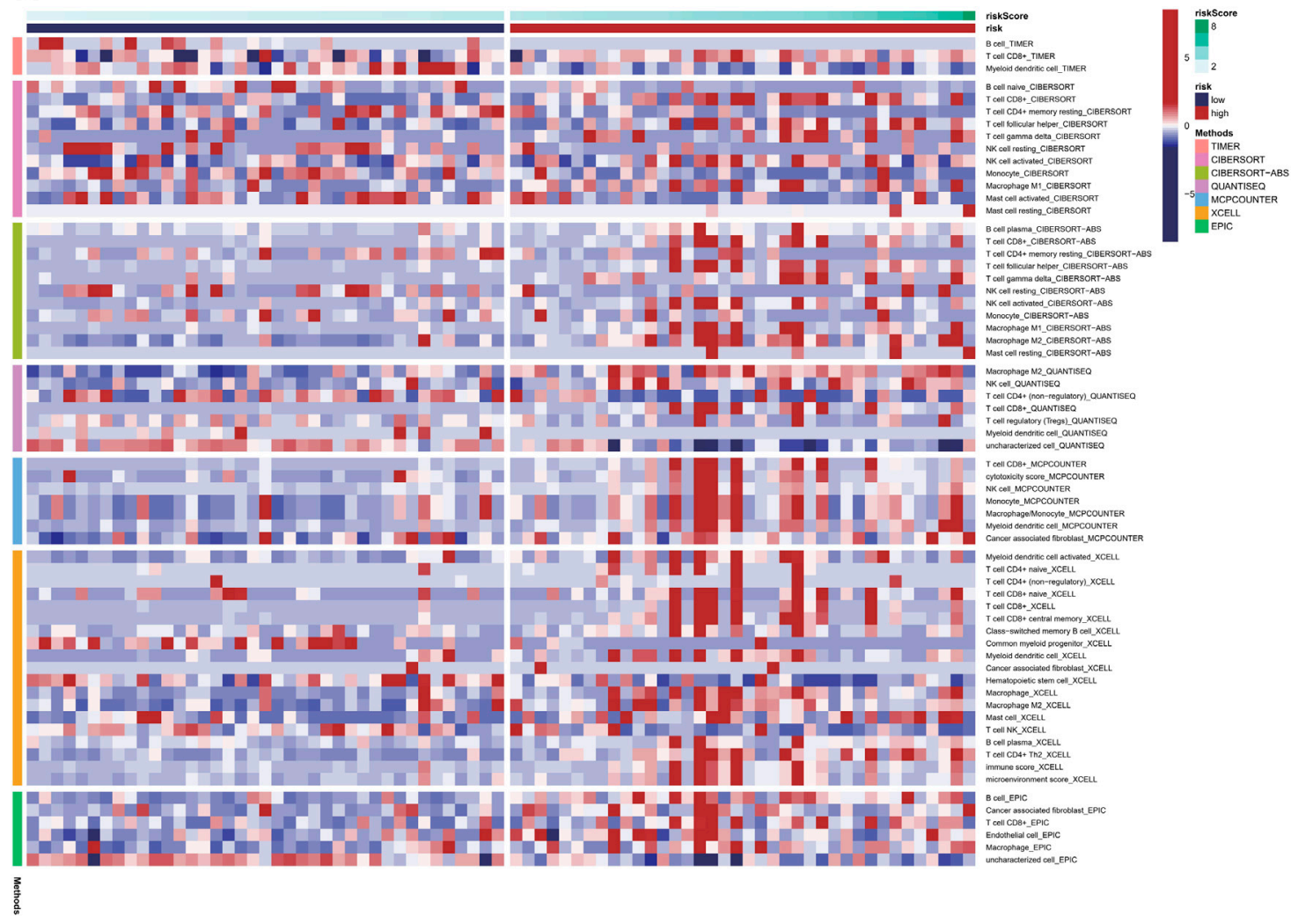

B Risk 官 low 官 high

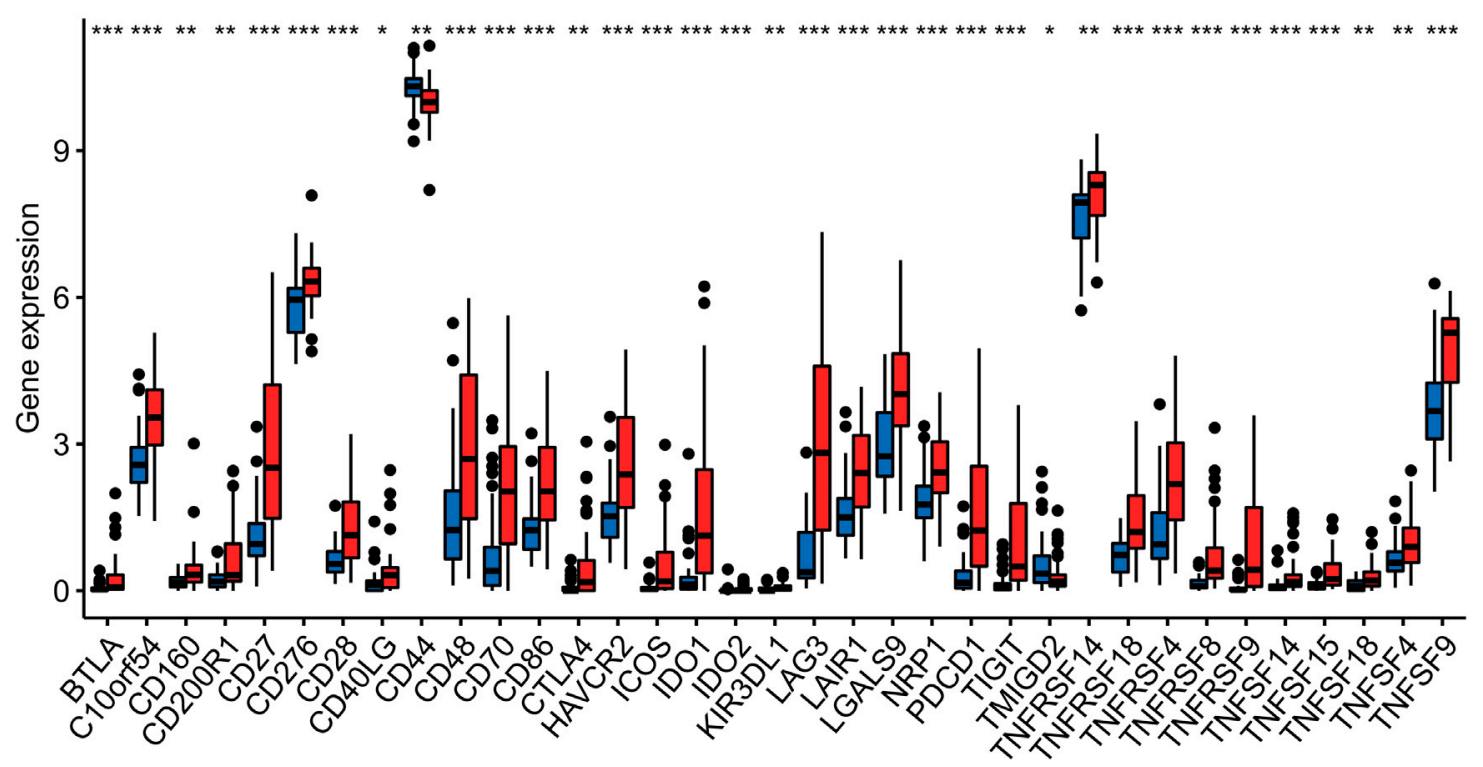

FIGURE 7 | Analysis of immune cell infiltration levels correlation analysis of immune checkpoint and in different risk groups. (A) The samples in the training cohort were displayed according to ascending risk scores, while the levels of immune infiltration in high-risk and low-risk groups were calculated by algorithm such as TIMER, CIBERSORT, Quantiseq and other methods, which were marked in different colors. The heatmap showed the level of each immune cell infiltration in the tumor microenvironment of the two groups of patients under different algorithms. (B) We could see that the high-risk group tended to have higher levels of immune cells in the tumor microenvironment. Likewise, The immune checkpoint genes were expressed differently in the two groups and gene tended to be higher in the high-risk group $\left({ }^{\star \star *} p<0.001\right)$. 


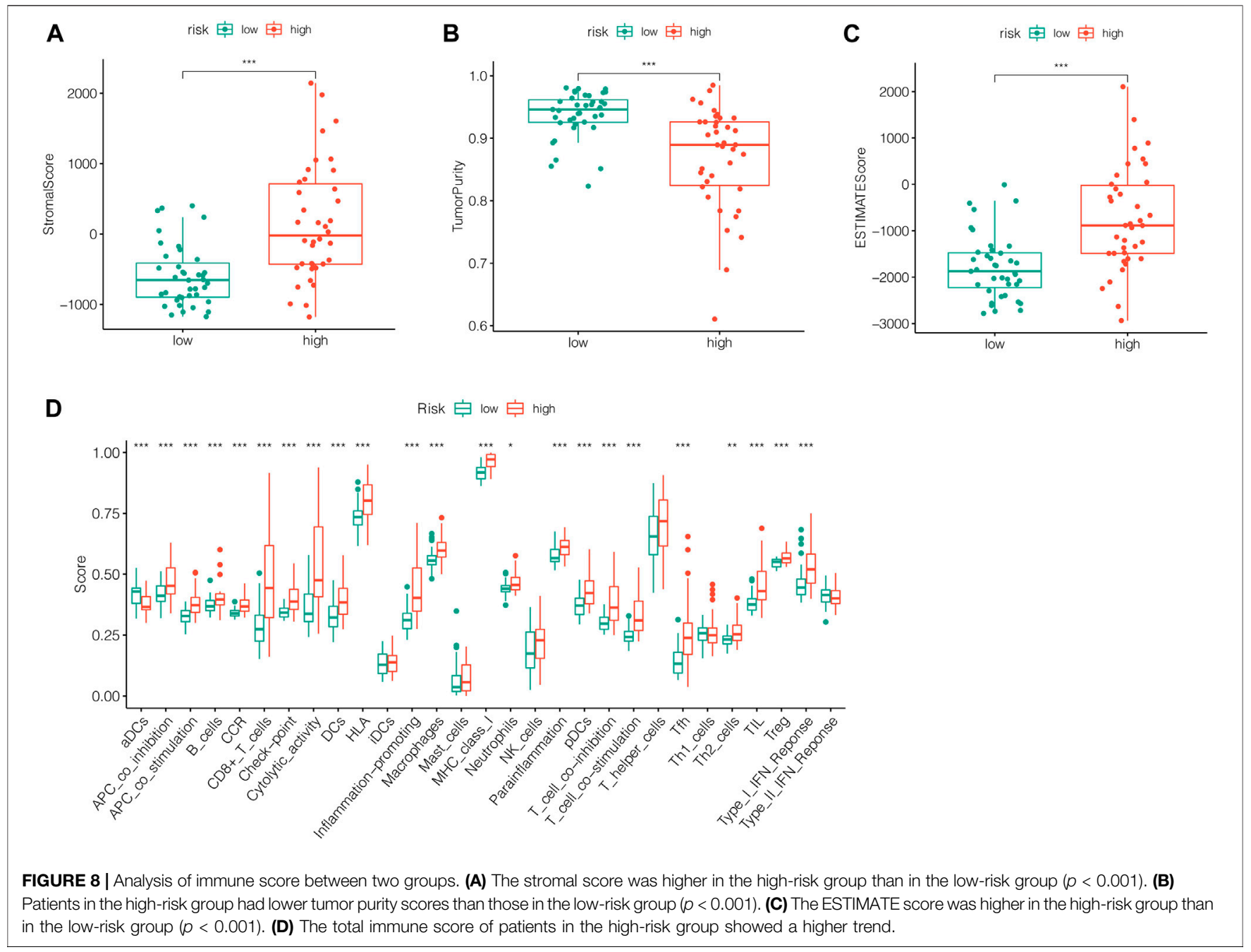

nuclear DNA synthesis, indicating the ANO6 gene may progress the proliferation of uveal melanoma cell lines (Figure 11B), which sheds light on further study. The results were statistically significant $(p<0.05)$.

\section{ANO6 Knockdown Attenuates Uveal Melanoma Cell Migration in vitro}

Scratch assays in Figure 11C showed that after the knockdown of ANO6 mRNA, MUM2B cells migrated slower than scrambled siRNA or mock-treated control cells, which means that ANO6 knockdown may attenuate the migration of uveal melanoma cells. The results were of statistical significance $(p<0.05)$.

\section{The Prognostic Value of This Pyroptosis-Related Gene Signature in Other Cancers}

Finally, we explored whether this signature also has prognostic significance in other tumors (gastric cancer, liver cancer, skin melanoma). The same method was used to calculate the risk scores of patients with gastric cancer, liver cancer and cutaneous melanoma in the TCGA database, and the patients were divided into the high-risk group and the low-risk group according to the median score value. Then we performed survival analysis between the two groups. As shown in Figures 12A-C, this signature has no prognostic significance in gastric cancer, liver cancer and skin melanoma. This indicates that the model has a certain degree of specificity for predicting the prognosis of UVM.

\section{DISCUSSION}

The idea that cell death is guided by internal instructions was first suggested in 1961 in a study of insects (Tower, 2015). This landmark discovery opens the door to the study of cell death. Since then, the concept of programmed cell death has been proposed, and its significance and mechanism are being intensively explored (Nagata and Tanaka, 2017). According to existing studies, cell apoptosis, cell necrosis, and cell pyroptosis constitute the three main parts of programmed death, and relevant studies are in full bloom (Bedoui et al., 2020). 

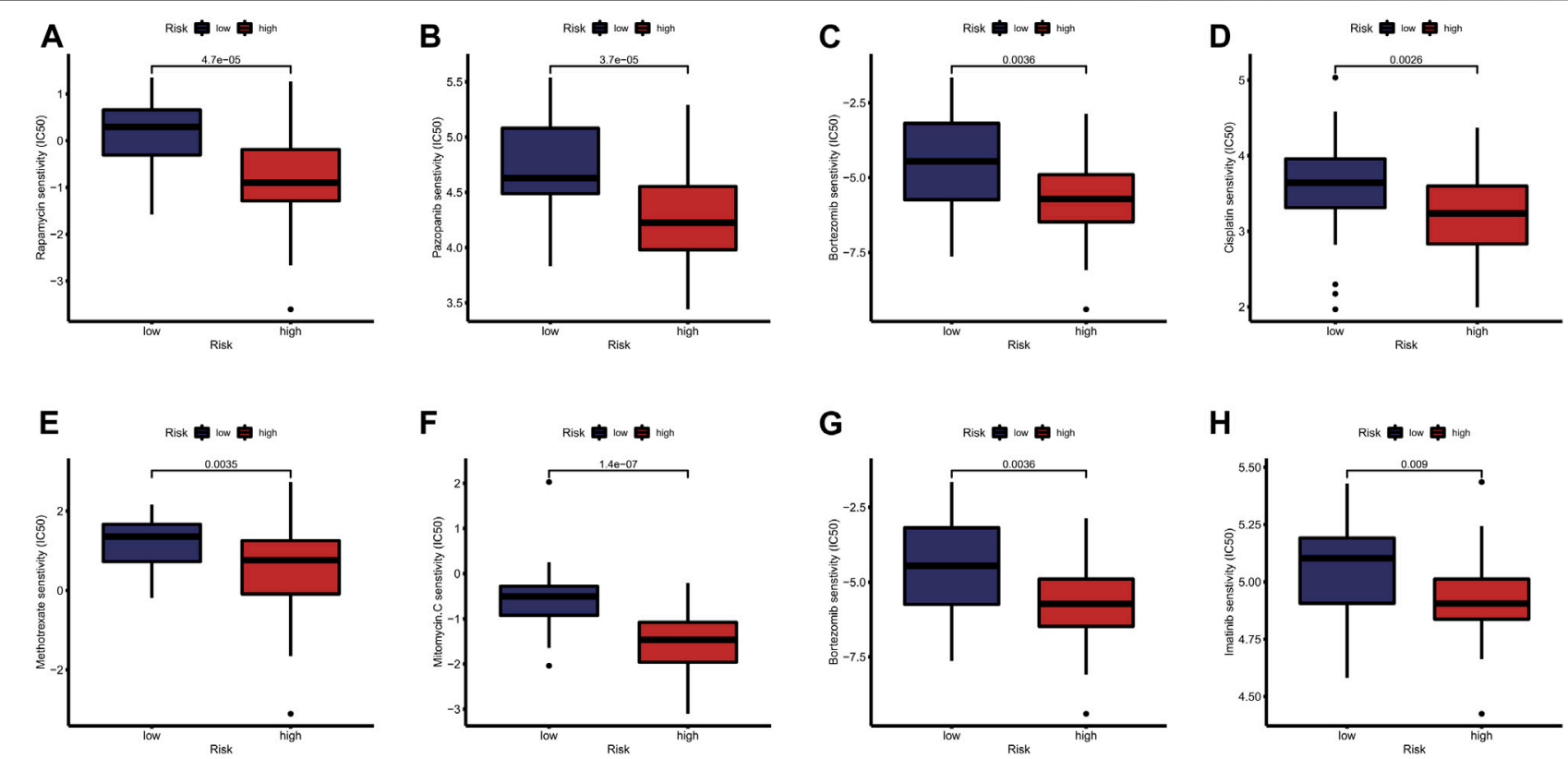

FIGURE 9 | Analysis of sensitivity difference of antitumor drugs in different groups. (A-H) The study of the sensitivity of different groups of patients to anti-tumor drugs can provide help for the formulation of future treatment regimens. Boxplot showed the differential $\mathrm{IC}_{50}$ of our previously established high-risk and low risk groups in the TCGA cohort. The high-risk group was more sensitive to antitumor drugs like Rapamycin, Pazopanib, Bortezomib, Cisplatin, Methotrexate, Mitomycin. C, Bortezomib and Imatinib. Those antitumor drugs were potentially more capable of inhibiting high-risk uveal melanoma with relatively minor dosage. However, further investigations are required to understand their mechanisms in Uveal Melanoma inhibition.

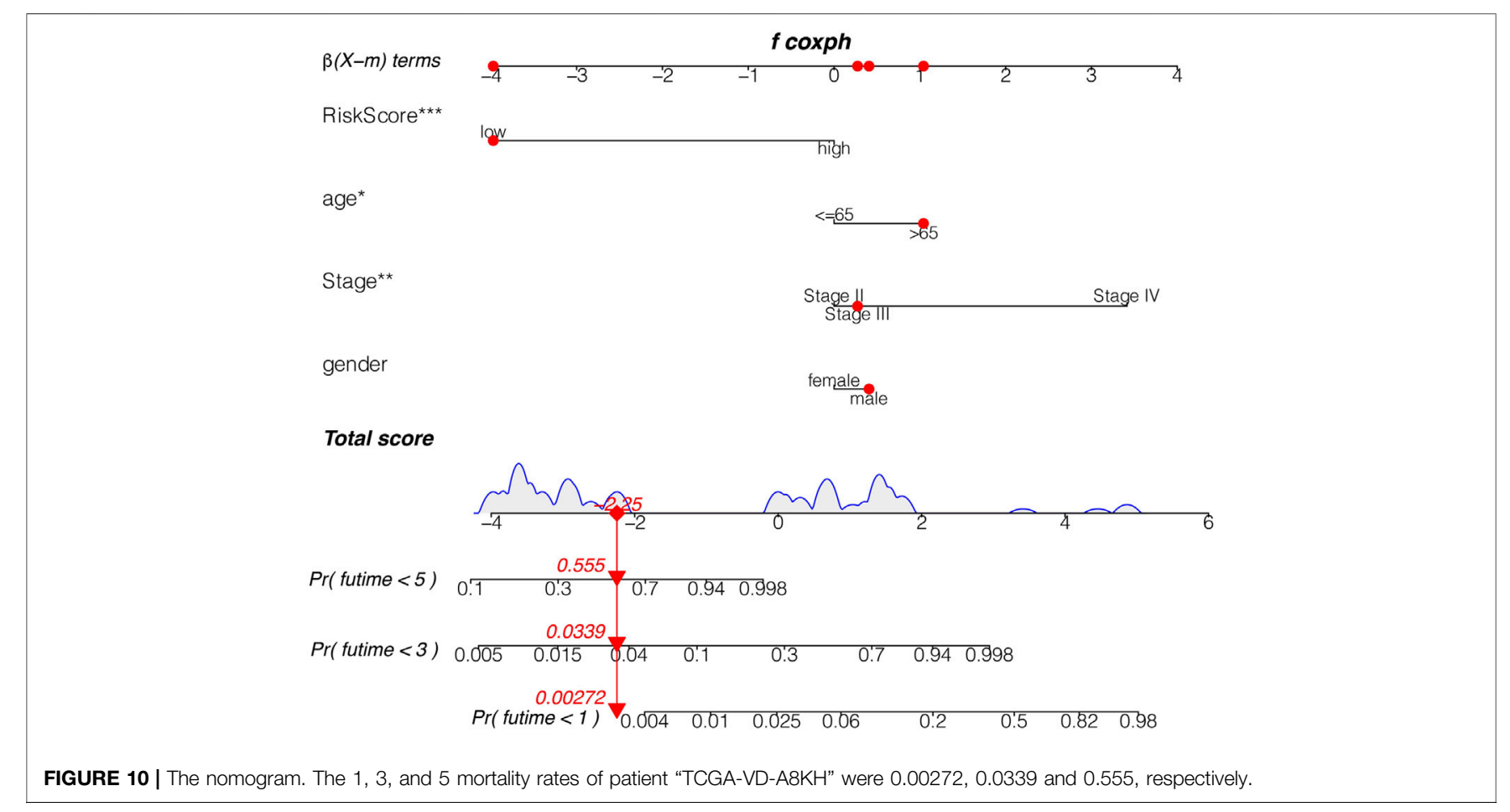

Uveal Melanoma, as one of the most harmful diseases to human vision, has seriously hindered the development of the social economy (Souto et al., 2019). Exploring the biological potential of this kind of cancer is primed and poised (Kashyap et al., 2016). Hallmarks of cancer mainly include excessive activation of growth signals, unrestricted replication, inhibition 
A Relative expression of ANO6 mRNA by $-2^{\Delta \Delta c t}$ method

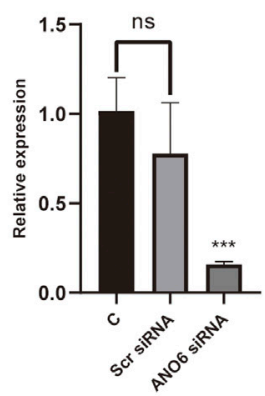

B
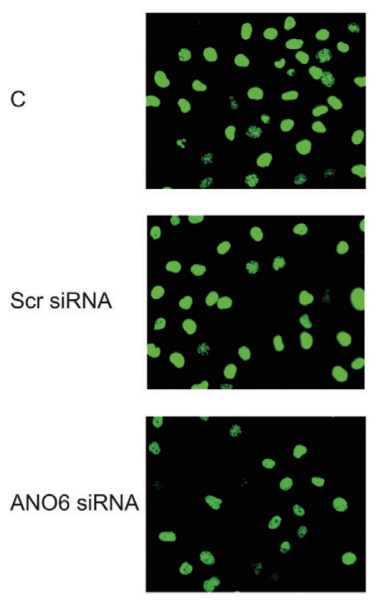

Oh

C

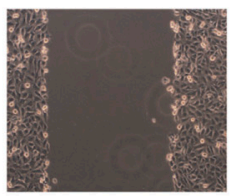

Scr

SiRNA

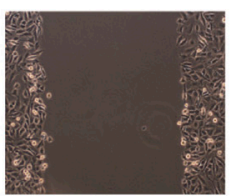

ANO6

SiRNA

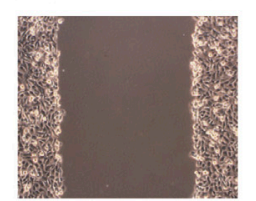

DAPI
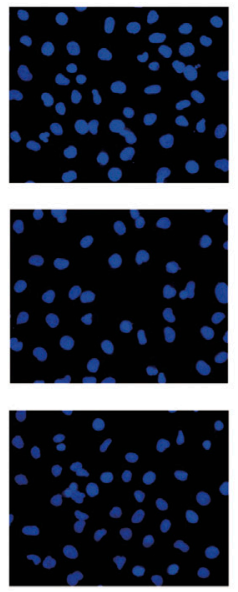

$6 h$
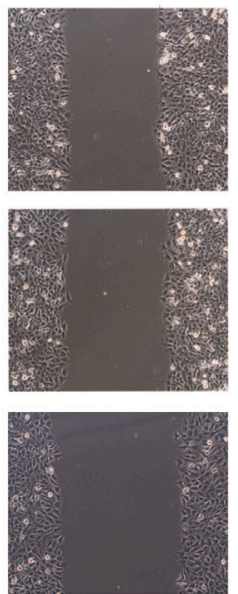

Merge
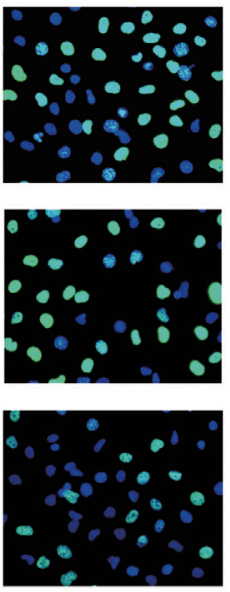

$12 \mathrm{~h}$
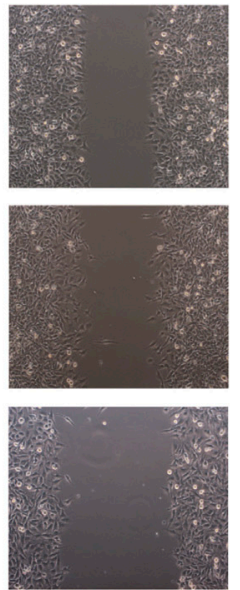

EDU Experiment

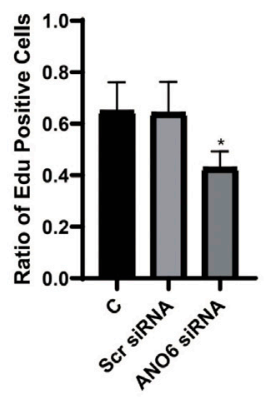

FIGURE 11 | Cell experiments of gene ANO6 to verify our results. (A) Firstly, RT-qPCR analysis was performed to validate the knockdown of gene ANO6 mRNA. The level of ANO6 mRNA expression was significantly downregulated after ANO6 siRNA transfection in MUM2B cell lines, which is valid for further investigation $(p<$ 0.001). (B) 5-ethynyl-2 deoxyuridine (EdU) assay was performed to test whether the knockdown of gene ANO6 could influence uveal melanoma cell proliferation in vitro. After knockdown of ANO6 gene, the in MUM2B cell lines showed a significant decrease in nuclear DNA synthesis, indicating the ANO6 gene may progress the proliferation of uveal melanoma cell lines, which sheds light on further study. The results were statistically significant $(p<0.05)$. (C) Scratch assays showed that after the knockdown of ANO6 mRNA, MUM2B cells migrated slower than scrambled siRNA or mock-treated control cells, which means that ANO6 knockdown may attenuate the migration of uveal melanoma cells. The results were of statistical significance $(p<0.05)$. 

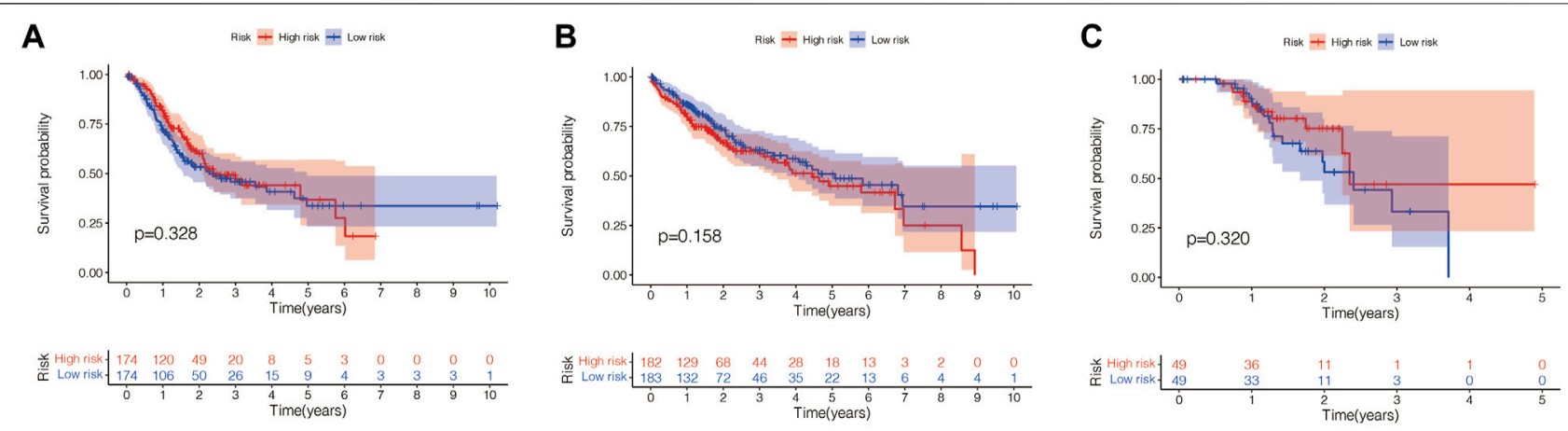

FIGURE 12 | The prognostic value of this pyroptosis-related gene signature in other cancers. $(\mathbf{A})$ This signature has no prognostic significance in gastric cancer $(p=$ 0.328). (B) This signature has no prognostic significance in liver cancer $(p=0.158)$. (C) This signature has no prognostic significance in skin melanoma ( $p=0.320)$

of cell death, immune re-editing, and metabolic reprogramming (Xie et al., 2021b; Xie et al., 2021c). As a kind of programmed death, cell pyroptosis plays an indelible role in both normal homeostasis regulation and cancer occurrence (Zhaolin et al., 2019). In cancer, pyroptosis has a dual effect. On the one hand, inducing pyroptosis of cancer cells can reduce tumor load in some cancers (Kesavardhana et al., 2020). Adversely, in other cancers, inflammatory substances released by cell rupture during pyroptosis can activate multiple cancer signals and may cause changes in the immune microenvironment that are beneficial for cancer proliferation (Hou et al., 2020). Hence, it's particularly important to explore the implications of char death in particular cancer types.

In this study, we built a prognosis model related to pyroptosis in UVM. According to the risk score, UVM patients can be divided into high-risk and low-risk groups, with differences in prognosis, immune infiltration, signaling pathways, and drug sensitivity between the two groups. It can be seen that our prognostic model can accurately assess the prognosis of UVM patients. In addition, we further verified our results in cell experiments.

At present, although multi-disciplinary and multi-protocol therapies have been applied to UVM, and the research on UVM is increasing, the understanding of the pathophysiology mechanism and tumor microenvironment of UVM is far from sufficient (Li et al., 2020). Moreover, the prognosis for UVM in the current context is still poor. Early diagnosis, early treatment, and the search for new prognostic markers are urgent (Zheng and Li, 2020). We constructed the prognosis model of UVM through pyroptosis-related genes and divided UVM patients into the highrisk group and low-risk group by calculating risk scores. Such a grouping pattern can accurately predict the prognosis of UVM patients. This is beneficial for the diagnosis and treatment of UVM.

The tumor immune microenvironment is still a research focus in oncology (Junttila and de Sauvage, 2013). In this complex environment, there are interactions between multiple cells and multiple signaling molecules to promote tumor growth (Wu and Dai, 2017). The degree of infiltration of immune cells in the tumor microenvironment provides a reference for us to understand tumor immunity and tumor pathogenesis (Kim and Bae, 2016). Inflammatory substances released by pyroptosis are involved in the formation of the immune microenvironment. It can be seen that after the prognosis model of UVM was constructed by pyroptosis-related genes, there was a difference in the degree of immune infiltration between the high-risk group and the low-risk group. This not only helps us to understand the immune regulatory role of pyroptosis in UVM but also provides a reference for us to explore the differences in the immune microenvironment of UVM.

Immune checkpoint inhibitors are a major discovery in the history of cancer therapy and have been successfully used in a variety of solid tumors (Dolladille et al., 2020). Cutaneous melanoma is a typical case. Although there are differences in the pathophysiology between UVM and cutaneous melanoma, the treatment of cutaneous melanoma can provide a reference for the treatment of UVM because they both originate from melanocytes (Alavi et al., 2018). Therefore, the significance of immunotherapy in UVM is also being explored in this study. We found that the high-risk group had a higher propensity to express genes associated with immune checkpoints than the low-risk group. This may suggest that UVM patients in the high-risk group may benefit more from immune checkpoint inhibitor therapy.

In our study, ANO6 is a significant gene in the pyroptosisrelated prognosis model. In addition, we verified the expression and functional effects of ANO6 in UVM through cell experiments. ANO6 has also been preliminarily described for its role in immunity. Szteyn et al. found that ANO6 is a Ca (2+) activated $\mathrm{Cl}(-)$ channel in mouse dendritic cells (DCs) and plays an important role in chemokine-induced DC migration (Szteyn et al., 2012). Ousingsawat et al. found that ANO6 is involved in bacterial phagocytosis and promotes THP1 macrophages to kill bacteria (Ousingsawat et al., 2015). Their study demonstrated the role of ANO6 in macrophage-associated immune defense. Seidel et al. found that ANO6 may be involved in the regulation of CD137/CD137L immune response and may have an impact on immunotherapy approaches targeting CD137 (Seidel et al., 2021). Therefore, ANO6 has potential significance in tumor immunity.

All in all, our study provides a new idea for the diagnosis, treatment, and prognosis of UVM. This has significant implications for UVM, a tumor with a poor prognosis. Future studies are expected to explore the tumor microenvironment of UVM. 


\section{CONCLUSION}

We constructed the prognosis model of pyroptosis related genes in UVM. Our model can accurately assess the prognosis of UVM patients. In addition, our model provides some ideas for exploring the tumor microenvironment of UVM.

\section{DATA AVAILABILITY STATEMENT}

The raw data supporting the conclusion of this article will be made available by the authors, without undue reservation.

\section{AUTHOR CONTRIBUTIONS}

YC and JX designed the study. LC and YH were involved in database search and statistical analyses. JX, YC, and MW were

\section{REFERENCES}

Alavi, S., Stewart, A. J., Kefford, R. F., Lim, S. Y., Shklovskaya, E., and Rizos, H. (2018). Interferon Signaling Is Frequently Downregulated in Melanoma. Front. Immunol. 9, 1414, 2018 . 2018 Jun 21. doi:10.3389/fimmu.2018.01414

Bedoui, S., Herold, M. J., and Strasser, A. (2020). Emerging Connectivity of Programmed Cell Death Pathways and its Physiological Implications. Nat. Rev. Mol. Cel Biol 21 (11), 678-695. doi:10.1038/s41580-0200270-8

Carvajal, R. D., Piperno-Neumann, S., Kapiteijn, E., Chapman, P. B., Frank, S., Joshua, A. M., et al. (2018). Selumetinib in Combination with Dacarbazine in Patients with Metastatic Uveal Melanoma: A Phase III, Multicenter, Randomized Trial (SUMIT). Jco 36 (12), 1232-1239. doi:10.1200/ jco.2017.74.1090

Chattopadhyay, C., Kim, D. W., Gombos, D. S., Oba, J., Qin, Y., Williams, M. D., et al. (2016). Uveal Melanoma: From Diagnosis to Treatment and the Science in between. Cancer 122 (15), 2299-2312. doi:10.1002/cncr.29727

Chen, X., Chen, H., Yao, H., Li, R., Zhao, H., Wang, X., et al. (2021). Turning up the Heat on Non-immunoreactive Tumors: Pyroptosis Influences the Tumor Immune Microenvironment in Bladder Cancer, [published online ahead of print, 2021 Sep 29]. Oncogene 29. doi:10.1038/s41388-021-02024-9

Chen, X., Chen, H., He, D., Cheng, Y., Zhu, Y., Xiao, M., et al. (2021). Analysis of Tumor Microenvironment Characteristics in Bladder Cancer: Implications for Immune Checkpoint Inhibitor Therapy. Front. Immunol. 12, 672158, 2021 . 2021 Apr 15. doi:10.3389/fimmu.2021.672158

Chen, X., Xu, R., He, D., Zhang, Y., Chen, H., Zhu, Y., et al. (2021). CD8+ T Effector and Immune Checkpoint Signatures Predict Prognosis and Responsiveness to Immunotherapy in Bladder Cancer. Oncogene 40, 6223-6234. doi:10.1038/ s41388-021-02019-6

Dolladille, C., Ederhy, S., Sassier, M., Cautela, J., Thuny, F., Cohen, A. A., et al. (2020). Immune Checkpoint Inhibitor Rechallenge after Immune-Related Adverse Events in Patients with Cancer. JAMA Oncol. 6 (6), 865-871. doi:10.1001/jamaoncol.2020.0726

Hou, J., Zhao, R., Xia, W., Chang, C.-W., You, Y., Hsu, J.-M., et al. (2020). PD-L1mediated Gasdermin C Expression Switches Apoptosis to Pyroptosis in Cancer Cells and Facilitates Tumour Necrosis. Nat. Cel Biol 22 (10), 1264-1275. doi:10.1038/s41556-020-0575-Z

Jager, M. J., Shields, C. L., Cebulla, C. M., Abdel-Rahman, M. H., Grossniklaus, H. E., Stern, M.-H., et al. (2020). Uveal Melanoma. Nat. Rev. Dis. Primers 6 (1), 24, 2020 . 2020 Apr 9. doi:10.1038/s41572-020-0158-0

Jiang, M., Qi, L., Li, L., and Li, Y. (2020). The Caspase-3/GSDME Signal Pathway as a Switch between Apoptosis and Pyroptosis in Cancer. Cell Death Discov. 6, 112, 2020 . 2020 Oct 28. doi:10.1038/s41420-020-00349-0 involved in the writing of manuscript and its critical revision. MW was responsible for the submission of the final version of the paper. All authors approved the final version. All authors agree to be accountable for all aspects of the work.

\section{ACKNOWLEDGMENTS}

We are very grateful for data provided by databases such as TCGA, GEO.

\section{SUPPLEMENTARY MATERIAL}

The Supplementary Material for this article can be found online at: https://www.frontiersin.org/articles/10.3389/fcell.2021.761350/ full\#supplementary-material

Junttila, M. R., and de Sauvage, F. J. (2013). Influence of Tumour Microenvironment Heterogeneity on Therapeutic Response. Nature 501 (7467), 346-354. doi:10.1038/nature12626

Kaliki, S., and Shields, C. L. (2017). Uveal Melanoma: Relatively Rare but Deadly Cancer. Eye 31 (2), 241-257. doi:10.1038/eye.2016.275

Kashyap, S., Meel, R., Singh, L., and Singh, M. (2016). Uveal Melanoma. Semin. Diagn. Pathol. 33 (3), 141-147. doi:10.1053/j.semdp.2015.10.005

Kesavardhana, S., Malireddi, R. K. S., and Kanneganti, T.-D. (2020). Caspases in Cell Death, Inflammation, and Pyroptosis. Annu. Rev. Immunol. 38, 567-595. doi:10.1146/annurev-immunol-073119-095439

Kim, J., and Bae, J. S. (2016). Tumor-Associated Macrophages and Neutrophils in Tumor Microenvironment. Mediators Inflamm. 2016, 6058147. doi:10.1155/ 2016/6058147

Kovacs, S. B., and Miao, E. A. (2017). Gasdermins: Effectors of Pyroptosis. Trends Cel Biol. 27 (9), 673-684. doi:10.1016/j.tcb.2017.05.005

Li, L., Li, Y., and Bai, Y. (2020). Role of GSDMB in Pyroptosis and Cancer. Cmar Vol. 12, 3033-3043. Published 2020 Apr 30. doi:10.2147/cmar.s246948

Luke, J. J., Olson, D. J., Allred, J. B., Strand, C. A., Bao, R., Zha, Y., et al. (2020). Randomized Phase II Trial and Tumor Mutational Spectrum Analysis from Cabozantinib versus Chemotherapy in Metastatic Uveal Melanoma (Alliance A091201). Clin. Cancer Res. 26 (4), 804-811. doi:10.1158/1078-0432.ccr-191223

Nagata, S., and Tanaka, M. (2017). Programmed Cell Death and the Immune System. Nat. Rev. Immunol. 17 (5), 333-340. doi:10.1038/nri.2016.153

Ousingsawat, J., Wanitchakool, P., Kmit, A., Romao, A. M., Jantarajit, W., Schreiber, R., et al. (2015). Anoctamin 6 Mediates Effects Essential for Innate Immunity Downstream of P2X7 Receptors in Macrophages. Nat. Commun. 6, 6245, 2015 . 2015 Feb 5. doi:10.1038/ncomms 7245

Ruan, J., Wang, S., and Wang, J. (2020). Mechanism and Regulation of PyroptosisMediated in Cancer Cell Death. Chemico-Biological Interactions 323, 109052. doi:10.1016/j.cbi.2020.109052

Seidel, J., Leitzke, S., Ahrens, B., Sperrhacke, M., Bhakdi, S., and Reiss, K. (2021). Role of ADAM10 and ADAM17 in Regulating CD137 Function. Ijms 22 (5), 2730, 2021. Mar 8. doi:10.3390/ijms22052730

Smit, K. N., Jager, M. J., de Klein, A., and Kiliç, E. (2020). Uveal Melanoma: Towards a Molecular Understanding. Prog. Retin. Eye Res. 75, 100800. doi:10.1016/j.preteyeres.2019.100800

Souto, E. B., Zielinska, A., Luis, M., Carbone, C., Martins-Gomes, C., Souto, S. B., et al. (2019). Uveal Melanoma: Physiopathology and New In Situ-specific Therapies. Cancer Chemother. Pharmacol. 84 (1), 15-32. doi:10.1007/s00280-019-03860-Z

Szteyn, K., Schmid, E., Nurbaeva, M. K., Yang, W., Münzer, P., Kunzelmann, K., et al. (2012). Expression and Functional Significance of the Ca2+-Activated ClChannel ANO6 in Dendritic Cells. Cell Physiol Biochem 30 (5), 1319-1332. doi: $10.1159 / 000343321$ 
Tang, R., Xu, J., Zhang, B., Liu, J., Liang, C., Hua, J., et al. (2020). Ferroptosis, Necroptosis, and Pyroptosis in Anticancer Immunity. J. Hematol. Oncol. 13 (1), 110, 2020 . 2020 Aug 10. doi:10.1186/s13045-020-00946-7

Tower, J. (2015). Programmed Cell Death in Aging. Ageing Res. Rev. 23 (Pt A), 90-100. doi:10.1016/j.arr.2015.04.002

Wu, T., and Dai, Y. (2017). Tumor Microenvironment and Therapeutic Response. Cancer Lett. 387, 61-68. doi:10.1016/j.canlet.2016.01.043

Xia, X., Wang, X., Cheng, Z., Qin, W., Lei, L., Jiang, J., et al. (2019). The Role of Pyroptosis in Cancer: Pro-cancer or Pro-"host". Cell Death Dis 10 (9), 650, 2019 . 2019 Sep 9. doi:10.1038/s41419-019-1883-8

Xie, J., Li, H., Chen, L., Cao, Y., Hu, Y., Zhu, Z., et al. (2021). A Novel Pyroptosis-Related lncRNA Signature for Predicting the Prognosis of Skin Cutaneous Melanoma. Ijgm 14, 6517-6527. 2021 Oct 8. doi:10.2147/ ijgm.s335396

Xie, J., Ruan, S., Zhu, Z., Wang, M., Cao, Y., Ou, M., et al. (2021). Database Mining Analysis Revealed the Role of the Putative $\mathrm{H}+$ /sugar Transporter Solute Carrier Family 45 in Skin Cutaneous Melanoma. Channels 15 (1), 496-506. doi:10.1080/19336950.2021.1956226

Xie, J., Zhu, Z., Cao, Y., Ruan, S., Wang, M., and Shi, J. (2021). Solute Carrier Transporter Superfamily Member SLC16A1 Is a Potential Prognostic Biomarker and Associated with Immune Infiltration in Skin Cutaneous Melanoma. Channels 15 (1), 483-495. doi:10.1080/ 19336950.2021 .1953322
Zhaolin, Z., Guohua, L., Shiyuan, W., and Zuo, W. (2019). Role of Pyroptosis in Cardiovascular Disease. Cell Prolif 52 (2), e12563. doi:10.1111/cpr.12563

Zheng, Z., and Li, G. (2020). Mechanisms and Therapeutic Regulation of Pyroptosis in Inflammatory Diseases and Cancer. Ijms 21 (4), 1456, 2020 . 2020 Feb 20. doi:10.3390/ijms21041456

Conflict of Interest: The authors declare that the research was conducted in the absence of any commercial or financial relationships that could be construed as a potential conflict of interest.

Publisher's Note: All claims expressed in this article are solely those of the authors and do not necessarily represent those of their affiliated organizations, or those of the publisher, the editors and the reviewers. Any product that may be evaluated in this article, or claim that may be made by its manufacturer, is not guaranteed or endorsed by the publisher.

Copyright (C) 2021 Cao, Xie, Chen, Hu, Zhai, Yuan, Suo, Shen, Ye, Li, Gong, Dong, Bao, Li and Wang. This is an open-access article distributed under the terms of the Creative Commons Attribution License (CC BY). The use, distribution or reproduction in other forums is permitted, provided the original author(s) and the copyright owner(s) are credited and that the original publication in this journal is cited, in accordance with accepted academic practice. No use, distribution or reproduction is permitted which does not comply with these terms. 